\title{
A Needs Assessment of Virtual Career Practitioners
}

\author{
Erica Fae Thomson - McMaster University \\ Bennett King-Nyberg- University of Victoria \\ Janet Morris-Reade- ASPECT BC \\ Cassie Taylor- Life Strategies Ltd \\ Roberta Borgen (Neault)- Life Strategies Ltd \& University \\ of British Columbia
}

\section{Abstract}

Like many other professionals, career development practitioners (CDPs) in British Columbia (BC) were forced to transition their services to virtual delivery at the beginning of the COVID-19 pandemic. In 2012, a BC Centre for Employment Excellence sponsored study found that among various delivery methods, virtual services were least preferred by practitioners (Neault \& Pickerell, 2013). The rapid shift to virtual work in 2020, unsurprisingly, left CDPs uneasy, unprepared, and unaware of how best to move forward. This research conducted a needs assessment of CDPs through a comprehensive survey based on The National Competency Profile for Career Development Professionals (Canadian Career Development Foundation [CCDF], 2021) and nine focus groups with practitioners working with underrepresented populations in the workforce. We found numerous areas of challenge for practitioners of all demographics. This report identifies which areas and competencies of service delivery have become easier for CDPs since the move to virtual services, and which areas

have become harder, supporting survey results with focus group conversations.

Keywords: virtual service, competency, needs assessment, challenges

\section{In 2020, the COVID-19} pandemic abruptly shifted many occupations, including those in the career development sector, to virtual ${ }^{1}$ or remote work in hopes of continuing to provide client services while protecting both clients and workers. The shift to virtual service delivery was unanticipated and immediate, leaving service delivery workers to quickly replace face-to-face services with virtual in an unprecedented time of need. Throughout our study we focus on these methods of virtual service delivery that were necessitated out of emergency circumstances, including intentional ways of reaching out to clients such as phone calls, emails, online platforms, and subsequently creating online

1 Virtual work is also described in this paper as online work/service delivery; although they are different entities, our participants referred to them synonymously, and therefore so do we.
\end{abstract}

modules. Although some organizations and individuals had previously established virtual programs and services, for many others the lockdown necessitated some replacements or adaptations of traditional in-person services. These changes affected various social service fields, and many service providers began to work exclusively from home or in office spaces that allowed little to no in-person interactions with clients or colleagues. This move to virtual work continues to create unique challenges for the career development sector and career development practitioners (CDPs), as the long-term effects of COVID-19 on the labour market will likely be severe (Akkermans et al., 2020). Beyond the unemployment issues exacerbated by the pandemic, many individuals are transitioning between occupations, sectors, or regions during the pandemic (Organisation for Economic Co-operation and Development [OECD], 2020). Many of the individuals now considering shifts in their careers would benefit from the expertise of CDPs to support career decision-making, planning, and implementation. The effects 
of this pandemic on a shifting labour market have created a "career-shock" that will have lasting impacts both locally and globally (Akkermans et al., 2020). CDPs play a large part in mitigating the impact of these current and anticipated changes (OECD, 2020), and career development services play a crucial role in solving the problems of workplaces in the emerging labour market (Luken, 2019). The OECD (2020) suggested that although some services may transition back to in-person work, digitization will continue to play a significant role in managing the anticipated influx of clients seeking CDP help. Trends in other industries suggest that remote and hybrid workforces will continue to increase (World Economic Forum [WEF], 2020). Additionally, some research suggests that developing competencies, such as those presented in The National Competency Profile for Career Development Professionals, along with improving resilience, may help to mitigate career shock (Akkermans et al., 2020).

\section{Research Questions}

This research project addressed, among others, one major question: Did the transition to virtual services make some skills/ responsibilities more difficult for CDPs, and if so, what were they? To identify these potential skills gaps, this research comprised a needs assessment of CDPs delivering services in British Columbia. Through a virtual survey and nine focus groups with CDPs assisting underserved populations, this research identified the skills gaps that emerged or had been exacerbated during virtual service delivery. As labour markets move into the pandemic recovery stage, having both CDPs and clients learn and understand how to navigate the virtual world is critical if we are to meet the needs of the future world of work. As well, the implications of the pandemic may shift over time (Akkermans et al., 2020), indicating the necessity to have a plan and strong partnerships to mitigate these possible struggles and barriers.

\section{Pre-Pandemic CDP Work}

CDPs are not immune to the general challenges of everyday work in the 21 st century, and virtual work has likely augmented and exacerbated pre-existing challenges for CDPs. The daily challenges that CDPs face transcend in-person service into the virtual world. The 2019 CERIC Survey of Career Service Professionals identified the top three barriers to success for CDPs in Canada as heavy workload, insufficient time with clients/ students, and inadequate financial resources (CERIC, 2020). These challenges, as well as burnout and poor salary/income, were identified as the key issues causing career professionals to leave the field (CERIC, 2020). Considering that a strong post-pandemic economy and labour market will heavily rely on CDPs, and that some competencies are of particular importance to mitigate this shock and help clients through an unstable economy and labour market, identifying old and new areas of challenge for CDPs is crucial for post-pandemic stabilization. Addressing these issues ensures that the career development sector can band together to implement programs to alleviate any further challenges in the sector and sustain an increased workload.

\section{Helping Skills and Mental Health}

Despite the fact that CDPs are not therapists, counsellors, or psychologists, client-practitioner relationships are built through therapeutic methods such as active listening (Walters et al., 2014). However, a major source of challenge in hiring career service professionals is that "most candidates have limited counselling skills" (CERIC, 2020). As the realm of CDP work extends beyond career-related tasks and includes making impactful influences across many facets of clients' lives, helping skills - including active listening, reframing, and facilitating self-efficacy and self-confidence - are important in facilitating client-practitioner relationships and achieving evidence-based outcomes (CERIC, 2020; Walters et al., 2014). The competency of creating a client-practitioner relationship that works to "nurture a collaborative and trusting relationship" (Canadian Career Development Foundation $[\mathrm{CCDF}], 2021)$ is of utmost importance.

COVID-19 has facilitated an unprecedented change to the 
fabric of society, resulting in isolation, job loss, poverty, and bans on proximal mental health supports, such as gyms and places of worship. COVID-19 has produced an increased number of people suffering from various mental health issues, including depression and anxiety (Abbott, 2021). Subclinical health struggles, such as difficulty with sleeping and eating, increased alcohol consumption and substance use, and worsening of chronic health conditions, have also increased throughout the pandemic (Panchal et al., 2021). There have also been reports of increased rates of suicidal ideation and attempts amongst youth (Hill et al., 2021) and adults (Fortgang et al., 2021). This may have put an unprecedented strain on existing mental health services, leaving many individuals without direct mental health aid.

Forging a positive client-practitioner relationship is a priority through these increased challenges. Mental health and mental illnesses are part of the career development field, whether acknowledged or not, where CDPs address and support psychosocial wellbeing (Redekopp \& Huston, 2019). Although the distinction between CDPs and therapists/ counsellors is an appropriate one to make - and should be very clear - skills transference between the fields can be helpful. Helping skills, including active listening, reframing, motivational interviewing, and facilitating self-efficacy and self-confidence, are incredibly important to client outcomes; however, they already exist as a skills gap for many CDPs and CDP candidates (CERIC, 2020). Counselling-related helping skills are integral in facilitating positive practitioner-client relationships (Walters et al., 2014).

\section{Supporting Clients Virtually}

A recent meta-analysis on virtual mental health therapy has shown that in-person and virtual interventions did not significantly differ in outcomes (Batastini et al., 2021). Hames and colleagues (2020) found that therapeutic modalities offered online are effective but posit that the effectiveness is contingent on specific competence to perform online therapy (e.g., therapeutic skills). However, as was noted above, CDPs have less experience and training with counselling-related helping skills, as well as a greater range of job responsibilities outside of counselling.

Forging a client-practitioner relationship is an integral prerequisite to any successful client outcome, but is also difficult, and has mitigating factors to consider. Within the client-practitioner relationship, Neault (2002) wrote that, of the difficulties facing practitioners, encouraging and promoting optimism and hope is perhaps one of the most arduous. This is significant, as facilitating optimism and hope in the face of career challenge is a substantial predictor for both career success and job satisfaction (Neault, 2002; Walters et al., 2014). Yukl (2006) identified three key client needs within the client-practitioner relationship that are necessary to meet prior to addressing any other goals: inclusion, affection, and shared control. Building these perspectives is contingent on overcoming the natural feeling of vulnerability and apprehension felt by many clients, typically by creating a relationship built on mutual trust, and the feeling that practitioners are collaborative, rather than authoritative (Walters et al., 2014); creating a space where a client feels that they "matter" is important (Schlossberg, Lunch, \& Chickering, 1989). According to Amundson et al. (2009), mattering has three key components: visibility, offering of help, and active listening. According to Walters and colleagues (2014), mattering is often expressed not by verbal cues, but by non-verbal actions; clients can feel that they matter simply by being offered a drink or given the choice of which chair to sit in when arriving at the office. Moving to virtual services may make it difficult to show the client that the practitioner truly cares, as active listening is often non-verbal. However, it may also include verbal interactions that work well in person but become interruptive when on video or through the phone.

Walters, Shepard, and Mani (2014) identified major components of active listening, including skills that, although effective in person, can be distracting and interruptive in virtual settings. The absence of such components, however, can create challenges to forging a positive client-practitioner relationship and may ulti- 
CANADIAN

JOURNAL OF

CAREER

DEVELOPMENT
REVUE

CANADIENNE DE DÉVELOPPEMENT

DE CARRIĖRE mately derail productive conversations. Vocal segregates, such as "mm-hmm", can be distracting and interruptive during video and phone calls. Following skills, such as repeating what someone is saying back to them after they speak, can derail otherwise productive conversations online. Providing clients with a feeling of visibility through practitioner affect can be difficult remotely. Reframing in person often occurs in the middle of emotional rants or stories, where the CDP may have to interrupt negative thought patterns to reframe them. Reducing client emotional outbursts, reacting appropriately to physical behaviours, and recognizing physical stress reactions such as sweating or fidgeting, may become more difficult over video. As well, there are further challenges that would have been near impossible to identify prior to moving online - camera angles make a large difference in how people maintain eye contact with other individuals. Depending on how the webcam is situated, there can be a miscommunication with respect to how attentive the participants of the call are (Chan \& Au-Yeung, 2021). In order to create a positive and safe environment for the client to open up, and for the practitioner to move the conversations forward in positive, productive ways, a client-practitioner relationship must be forged. CDPs may already struggle with counselling-related helping skills (CERIC, 2020); moving client-practitioner interactions online may have exacerbated these gaps.

\section{Emerging CDP Roles and Responsibilities}

CDPs work with employers and stakeholders to create employment opportunities for clients in local contexts (CCDF, 2021). This may include working to address gaps in service, compiling lists of potential employers and employees, or initiating contact between employers and clients. CDPs need to support program developers in searching for and recruiting appropriate candidates and aiding in selection processes. Although the pandemic-related layoffs and shift to virtual services may have made community connections more difficult for CDPs to maintain, it is increasingly important that CDPs retain these relationships in order to support their clients (OECD, 2020). The COVID-19 pandemic will inevitably change labour markets and demands (OECD, 2020). According to the CCDF (2021), a CDP's role includes awareness of, and response to, these changes.

A transition to virtual work across many sectors means that CDPs may have to help clients with the digitization of their benefit plan systems or other work-related supports (OECD, 2020). CDPs must also be able to work with clients on various aspects of career development including creating resumes, communications, portfolios, and value propositions, securing good references, preparing for interviews, and establishing professional networks. CDPs must offer appropriate guidance tailored to their client's individual needs. As the types of available jobs shift, CDPs must be prepared to adjust their approach to working with clients. For example, CDPs may have to aid in preventing unemployment by guiding clients through new work agreements or terms, including an influx of shorttime work (OECD, 2020). They will have to encourage job seekers to actively look for work and provide constantly updated information and training (OECD, 2020). CDPs may also need to host more virtual job fairs, and tailor goals to target employment that is available during the pandemic (OECD, 2020).

Sampson et al. (2020) discussed the transition to virtual services and recommended applying a change management strategy that puts the needs of the client first, blending types of technology and media. They suggested that it is necessary to work with existing cultures to create learning environments that best meet the needs of the client. Further, they concluded that CDP skills must evolve to meet the demands of a virtual environment and that this evolution is necessary for clients to access the expertise of career professionals. Banks and colleagues (2020) found that challenges for workers in the virtual social care field included maintaining trust, dignity, and service user autonomy during remote work. Another challenge was providing specific, tailored services to fit each individual clients' needs. Banks and colleagues (2020) found that a large challenge for workers was allocating the limited resources at their disposal, and balancing the rights and needs 
of clients, practitioners, and other parties.

Virtual career development practice has potential limitations. Sampson et al. (2019) identified that virtual services may result in lower quality of assessments, lower quality of information, and various challenges with the technological abilities of both the practitioner and the client. Further, they acknowledged that CDPs themselves may not have the competencies to provide distance supports using technology. Sampson and colleagues (2020) concluded that CDP skills must evolve to meet the demands of a virtual environment and that this evolution is necessary for clients to access the expertise of career professionals. Additionally, Como et al. (2021), through a comprehensive review of relevant literature, identified that inadequate support for remote workers may cause a decline in mental and physical health if at-home working conditions are not ideal. The rapid switch to virtual service delivery, as well as added COVID-19 stress, complicates work-life wellness for both CDPs and the workers they help (Como et al., 2021). Como et al. (2021) highlighted that CDPs must continue to be aware of the implications of remote work when dealing with clients and that support must be maintained for both organizations and remote workers, including those working virtually. It is important to note that Neault and Pickerell (2013) had previously found that virtual services were the least preferred service delivery model for CDPs. Combining this with the novel challenges CDPs were facing during the COVID-19-related move to virtual services, indicated an urgent need for the current research.

\section{CDP Competencies}

The role of CDPs is complex, both in-person and in virtual service delivery. In order to create a solid foundation for the professionalization of the career development sector within Canada, a multi-year project has recently culminated in the new National Competency Profile for Career Development Professionals (2021). In this document, the Canadian Career Development Foundation (CCDF; 2021) identified 65 competencies across 16 series that are necessary for CDP success. These competencies are clustered into two sections: Professional Practice and CDP Characteristic. The delivery of these competencies through the work of dedicated CDPs is crucial for the future of Canadian job markets and economies (OECD, 2020). This new framework of competencies and skills will help to identify what the outcomes of CDP education, certification, and training should focus on, as well as provide a basis for evaluating the successes and needs of CDPs in BC and across Canada. These competencies come at a pivotal point for CDP workers, individuals, and organizations as the events of the global COVID-19 pandemic have forced many work operations to move fully or partially virtual.

The standardization of these competencies was occurring as the COVID-19 pandemic began; as such, there has been little to no research addressing these specific competencies. Research has also been limited on how the pandemic-related transition of CDP work to virtual environments has affected CDPs' ability to master and demonstrate these competencies. This study extends the literature by including the framework of these vital competencies. Through this lens, we were able to identify which actions related to $\mathrm{CDP}$ competencies pose the greatest challenge in providing virtual services, as well as which competencies CDPs self-identified as being undertrained in. Throughout this study we utilized the overall series of 16 , as well as the specific competencies within them, as a basis for our inquiries. These competencies became the foundation for the needs assessment survey in our research. We do not reference the competencies directly by name, as they were in their final stage of development at the time of our inquiries. However, we used them as the framework for our data collection and conversations.

\section{Materials and Methods}

Data collection was conducted in two phases, using a mixed quantitative and qualitative approach, comprised of a survey and subsequent focus group discussions. We employed an explanatory design of data collection to expand on our quantitative results with qualitative data collection (see Creswell \& Clark, 
2017 for further information on explanatory mixed-method data collection). This method allowed us to both follow-up on our quantitative results, as well as to select participants for qualitative data collection. Phase 1 of our data collection employed a custom-designed online survey of career and employment service providers in BC. Phase 2 comprised 12 focus groups to further explore the impact of the shift to virtual services on underrepresented populations in the workforce. These two complementary phases allowed us to gather comprehensive data from CDPs all over BC, as well as to focus on the unique barriers that exist in delivering virtual services to specific populations. It also allowed us to validate and expand on our survey results.

\section{Consultation}

Throughout the project, we consulted with a group of eight career development leaders who had been convened by ASPECT to form a Virtual Learning Consortium (VLC) to offer project guidance and add a validity check to each phase of the research. At our first meeting with the VLC, we presented them with our preliminary ideas for the research focus and process; they verified the direction we were taking and offered valuable insights that enriched our approach. With the support of the VLC and subsequent informal conversations with other members of ASPECT BC, we were able to condense an extensive draft of survey questions to a manageable number of items that could be reasonably completed within 10-15 minutes. The VLC also agreed to test both the survey and the focus group protocol; their insights and practical guidance helped to refine and restructure our questions to best address the needs of the sector.

\section{Survey Development}

Combining a broad literature review (keywords: career development, COVID-19, competencies) with the 26-competency framework from the Canadian Career Development Foundation (2021), we were able to create a focused list of survey questions and focus group topics. To further narrow down our questions we conducted a narrow literature review organized by the 26 competencies and compiled a list of potential questions based on this literature.

The survey tool was structured into two parts. Part 1 gathered basic demographic information such as gender identity, age, years of employment within the career development sector, and level of education. Part 2 was grounded in the new competency framework for Canadian CDPs (CCDF, 2021). This part assessed various competencies - such as "Building rapport and creating positive relationships with your clients." Participants rated each statement on the degree of change since their transition to virtual services, using a 5-point Likert scale. The scale consisted of $0=$ Not Applicable, 1 = Much Easier, 2 =
Somewhat Easier, $3=$ No Change, $4=$ Somewhat Harder, and $5=$ Much Harder.

\section{Focus Groups}

In addition to our survey, we conducted nine focus groups with providers who served various populations. These focus groups lasted between 60-90 minutes and were conducted via Zoom by the projects' research assistants. Focus groups were facilitated with a semi-structured interview guide which focused on the move to virtual services and were used as a method to validate, expand on, and confirm survey responses. Prompts centred on CDPs' individual experiences and thoughts surrounding the transition to virtual service delivery, as well as how they were able to effectively use the necessary competencies to serve groups of underrepresented clients. Questions during focus groups surfaced general thoughts about the transition to virtual services, as well as validating and expanding on survey-related results. For example, participants were asked their thoughts about the top five competencies that survey results indicated had become harder to perform with the shift to virtual services (see Figure 1).

\section{Participants}

\section{Survey}

Survey participants were recruited through ASPECT BC's members (e.g., notices in weekly newsletters, mass emails to mail 
list members) and social media posts (i.e., Facebook, Instagram, and LinkedIn); our aim was to achieve broad representation of CDPs from across British Columbia. Participants in the survey were asked to select which underserved populations, if any, they worked with for more than $50 \%$ of the time. Categories of underserved populations included: immigrants, newcomers, and refugees; older workers; persons with disabilities or with mental health or addiction challenges; people from rural, remote, and northern communities; people with essential skills gaps; adults without post-secondary education; racialized individuals; those fleeing domestic violence; veterans; women; and youth. After comprehensive data cleaning to remove bots and incomplete survey responses, we were left with 179 respondents $(\mathrm{N}=179)$.

\section{Focus Group}

Focus group participants were also recruited through ASPECT BC's members and social media posts, as well as from responses to the survey question asking which underserved populations they worked with, snowball methods, and reaching out to ASPECT's contacts. Those opting to participate in the focus groups were asked to volunteer for a maximum of two groups based on the populations that they specialized in. Each focus group had 3 - 10 participants and, in total, included 58 CDPs from various locations across British Columbia. Nine focus groups with CDPs who deliver services to a wide variety of clients were conducted.

\section{Data Analysis}

\section{Survey}

During our survey data collection via Microsoft Forms we were overrun by bots that began responding to our survey in swarms. As research assistants were regularly monitoring survey responses, the bot attacks were noticed within a few hours. The research assistants immediately identified patterns in the bots' responses that made it relatively easy to identify and eliminate them; typical bot responses including selecting a province of service delivery outside of British Columbia, leaving the majority of questions blank, or providing irrelevant job titles (i.e., titles not related to the career development sector). Through these criteria and more (see King-Nyberg et al., 2021) we were able to eliminate bot responses from the survey results. Excel, Python, and the Statistical Package for the Social Sciences (SPSS) were used for statistical analysis. We ran comparison of means tests (e.g., t-test, analysis of variance) on demographic information and average Likert score.

\section{Focus Groups}

The qualitative analysis was conducted by our two research assistants and ASPECT's CEO; it comprised a thematic analysis of focus group recordings. Each recording was examined by at least two researchers; themes were validated and coding discrepancies were resolved by a third researcher. After identifying major themes in the data (e.g., technology challenges, mental health) relevant quotations were extracted from the recordings and logged. These quotes became the backbone of our qualitative analysis as they illuminated the experiences of CDPs and gave context to the survey results.

\section{Results}

\section{Descriptive Statistics}

Approximately $78 \%$ of participants identified their gender identity as woman, $21 \%$ as man, and $1 \%$ as non-binary. All of the participants worked primarily with clients based in BC - the targeted province for this study. The majority of participants were between the ages of 45-54 (33\%), $35-44(28 \%)$, or 55-64 (23\%). A smaller subset of participants were between the ages of 25-34 (10\%), $18-24(3 \%)$, or $65+(3 \%)$. Most participants $(45 \%)$ had worked in the career development sector for $10+$ years. Interestingly, the next largest number of participants (31\%) were relatively new to the sector, having worked in it for only $1-5$ years; $23 \%$ had worked in the sector for between 5-10 years, and $9 \%$ for less than 1 year. Most participants reported post-secondary education, with either a Bachelor's degree $(32 \%)$ or a college diploma (27\%).

Participants were almost equally divided in terms of 
completion of a career development-related training program, with 51\% reporting "yes" and 49\% reporting "no." Participants represented a wide range of roles and titles including "Job Developer," "Employment and Client Coordinator," "Employment Advisor," and "Career Educator," among many others. Practitioners also listed the groups that they worked with (not mutually exclusive). The fewest practitioners selected that they work with veterans (60), refugees (69), and people from rural, remote, and northern communities (98). The populations most often worked with were women (148), persons with disabilities (144), and Indigenous peoples (144).

\section{Likert Results}

After averaging each participant's responses across Likert questions, we found that the distribution of means were somewhat normal; other measures of central tendency (i.e., median and mode) were close to 3.00 (M $=3.049$, $\mathrm{SD}=0.67)$. However, although the modal response was 3 , the next common response was more often 4 than 2 , showing a slight negative skew towards more difficult. Each question we asked garnered at least some responses that indicated things had become more difficult since moving to virtual, and at least some responses that indicated things had become easier, clearly indicating that the move to virtual services had not been a one-size-fits-all experience. Figure 2 shows the results of the most relevant Likert questions, ordered from greatest to least frequency of "Harder" responses. Although there were 30 Likert questions asked on the survey, for this article we chose to report on only the items that became quite a bit harder; the natural partitioning for this was after the tenth item.

Although we tested for variability within demographic groups (e.g., age, gender identity, level of education), we found no statistically significant between-group differences in overall Likert scores. This suggests that our results could be broadly generalizable across all demographic categories of CDPs, providing avenues for intervention and training that could benefit the majority of service providers.

\section{Survey and Focus Group Results}

The results presented in Figure 1 support our hypotheses that had been formulated from the literature review and VLC consultants - that specific areas of career development are important and have likely changed in difficulty during the shift to online services. Of competencies perceived as more difficult since the move to virtual services, 4 of the top 10 (i.e., engaging with reluctant clients, building rapport and creating positive relationships with your clients, addressing client's health, and reducing cultural challenges in communication or ideas) are directly related to the necessity of having good virtual communication and relationship-building skills. While there were conflicting opinions on how virtual services lends itself to creating positive relationships and building rapport with their clients, a common theme was that, despite the increased challenges of virtual service delivery, virtual services were beneficial for creating and maintaining bonds with clients due to increased ease and frequency of contact.

The good side of the whole virtual service is that I noticed that I created, I dare to say, quite a strong bond with my clients. It feels like they are more in arms reach. Before it was an email, "Let's schedule an appointment." A phone call, "When are you available?," and then you wait for that day to talk to them. Now that we don't know when we'll be able to see each other again it's a phone call and we talk about everything. Meet me on Zoom, and we talk about everything. Today, or tomorrow. It became a closer connection, I think. Yeah, it was bittersweet. There's the good and the bad.

Others echoed the ease of timely connection that virtual and remote service brings. However, they expanded to touch on a point that was made in all focus groups: telecommunication, even with video, made it incredibly difficult to read clients' non-verbal cues and body language.

Of course, it's easier just to hop on the phone, or get on a kind of video conference. But ... you 
Figure 1

\section{Likert Statements}

Engaging with reluctant clients

Helping clients with accessibility (e.g., digital literacy, transportation)

Building rapport and creating positive relationships with your clients

Addressing client's health (e.g, negative health behaviours)

Reducing cultural challenges in communication or ideas

Recruiting participants for programs, service, or training opportunities

Establishing and sustaining professional relationships

with members of the community and relevant associations

Allocating limited resources (e.g., physical, virtual, and emotional resources) ethically

Aiding clients in job readiness (e.g., resume and cover letter writing, interviews, handshaking)

Facilitating group learning and training

Separating personal life from work

Discussing and assessing pre-employability factors/ client challenges (e.g. mental health)

Identifying client strengths and weaknesses

Customizing your approach to client learning and employment

Communicating well and maintaining positive relationships with your colleagues

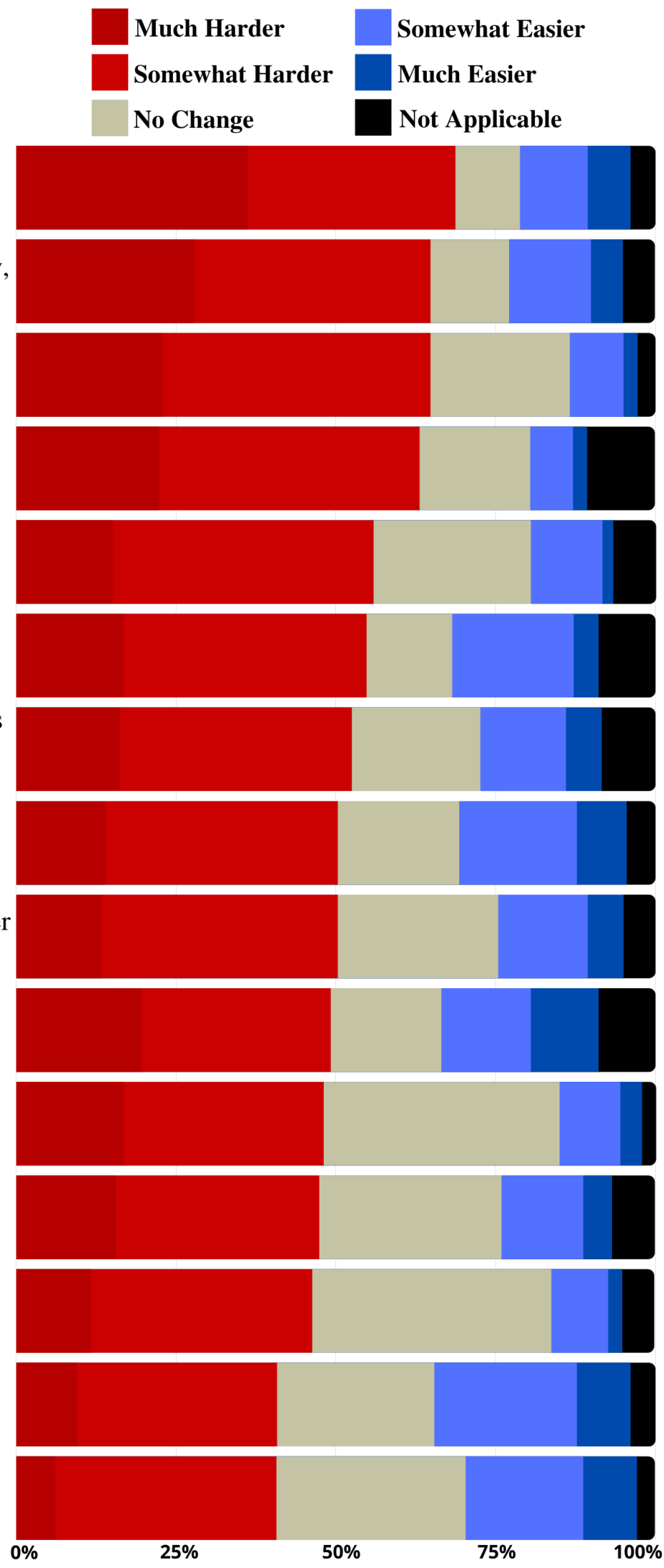


lose some of the message delivered from the communication, because you only see the headshots, right? The whole body language is invisible. So, some of the gestures, or some of the non-verbal cues, it's impossible for you to observe. So, that's the challenge part.

Additionally, some focus groups noted that it was more difficult to see clients' emotional responses. For example, it was more difficult to see shaking hands, or nervous fidgeting that may be indicative of anxiety. Body language was a focus in many of our focus groups, noting that these non-verbal cues are especially important to serving clients with barriers, such as disabilities, language barriers, or those in dangerous home situations. In order to mitigate the level of communication that was lost with virtual services, some participants noted that they needed to become over-animated and elevate their expressions to make sure their welcoming nature translated through the screen. They increased the use of inflection and animation in their voice and made larger arm gestures to indicate their enthusiasm and connection. Despite actively trying to reduce any amount of lost connection, some found that the learning experience was just not the same.

However, in the absence of our in-person services, we felt that as much as we tried, the learning experience was not to the same effect as it would have been in-person, especially for the participants in need of developing social skills, soft skills, and confidence-building. So, there was a huge disadvantage. So in a nutshell I can't tell if it works for us or not; it's a bit of a complicated question.

Focus group participants reported that virtual service delivery brought some big disadvantages for strengthening some necessary skills in their clients. Many said that the skills likely could be taught over video, but felt that they were woefully undertrained to do so. Overall, those represented in the survey and focus groups struggled with creating connections through virtual settings. Interestingly, engaging with reluctant clients was brought up less often in the focus groups than was building a good relationship with the client. When prompted to further expand their feedback, participants explained that engaging with reluctant clients was an issue that could only be solved by being able to communicate effectively and motivate, which was only possible after creating a positive relationship. Focus group participants reported that within virtual service delivery, they had a lack of skills in this foundational area, which prevented them from resolving their clients' other issues.

Two of the statements in the survey (i.e., helping clients with accessibility and aiding clients in job readiness) relate to preparing clients for work. Participants reported that helping clients with their own digital literacy skills and facilitating clients' job readiness was more difficult to do without a physical, in-person, hands-on approach. On the other hand, focus group participants identified one positive, geographic aspect of virtual services on client accessibility; many individuals who had access to adequate technology and stable Internet connections had the opportunity to access services from their homes, avoiding time-consuming and costly trips to CDP offices. This applied both to individuals in rural and remote communities and to those who lived within a city. As one CDP remarked:

All of [a] sudden they were able to take [programs] virtually rather than having to try and figure out a way to get to [the city] from living up in like [a farther away city] or something like that. Pros and cons with it for our program. It did make our services more accessible, geographically, because we operate out of [local cities]. We were able to accept participants from other regions. So, that was a good thing in that sense.

However, they also noted that these advantages were not universal. Many rural and remote regions had inadequate and inconsistent access to Internet or cellular services, and many clients could not access or use a computer properly. Focus group participants often spoke about the large number of individuals who had barriers to technology (e.g., people who were homeless, refugees, immigrants, or elderly), which hindered accessibility to services and the 
ability for clients to acquire the necessary skills to increase their job-readiness.

The survey also identified increased difficulty in client recruitment (i.e., recruiting participants for programs, service, or training opportunities) and engagement (i.e., facilitating group learning and training) since the move to virtual service delivery. Despite these issues being common amongst the study participants, it was interesting to note demographic between-group differences. For example, many participants reported that youth were difficult to recruit and, once in the program, would typically have their microphones muted and video camera off during sessions, preferring text-based interactions on their phones. Other demographic groups (e.g., women fleeing violence; refugees) expressed concern about their photographs or videos being shared or recorded, or about household members overhearing their virtual sessions.

Survey respondents also indicated increased difficulty after the shift to virtual services with their communication with, and connection to, relevant community resources and associations. As further discussion with focus group members clarified, much of the job placement that typically occurs is through direct, friendly interactions between a CDP and a leader of an organization, business, or community association. One participant shared a story of how many of her placements were from casual conversations with franchise and small business managers who would find odd jobs that could be done with minimal formal credentials. The pandemic prevented many of those interactions from occurring.

Finally, many CDPs found it more difficult to distribute resources ethically and fairly (i.e., allocating limited resources), reporting that they struggled to stretch their limited resources in ways that would adequately reach the clients who most needed them.

\section{Discussion}

Overall, the survey and focus group results confirmed previous research surfaced in the literature review (see Kettunen \& Sampson, 2018; Sampson et al., 2020): there are specific areas of career development that have become more challenging since the move to virtual services.

To improve client services, each of these areas of increased challenge could be targeted with specific training interventions for CDPs and/or structural interventions that will benefit clients themselves. A1though some of these interventions will need to be newly developed and target logistical difficulties (e.g., individuals who lack access to Internet, community engagement, and participant recruitment) others can be adapted and customized from a strong foundation of career development interventions that have been previously successful.

Recommended points of intervention here are at the level of enhanced training for CDPs. By focusing on the challenges that
CDPs themselves faced, organizations can implement training and supports that target the employees' specific needs. For example, CDPs consistently mentioned that the benefits of training in body language comprehension were important and effective for in-person interactions but was not useful virtually. Therefore, modified training on reading other non-verbal body language, such as voice tone, sentence and word tempo, rate of breathing, and eye movements may prove to be effective. Additionally, increased training on teletherapy skills may be an important method to provide CDPs with the confidence and skills they need to help clients. A common claim heard throughout the focus groups was that this kind of specific training (e.g., motivational interviewing, active listening) was especially helpful for building rapport and a good client-practitioner relationship. Training CDPs in motivational interviewing skills can also help aid them in the increased difficulty of confronting negative health behaviors. Remedies to logistical difficulties could include introducing a multi-faceted communication plan for practitioners and clients. This may include flexibility in length of sessions and method of communication (e.g., texting vs video-calling) and may also prove to help build relationships, engage reluctant clients, and reduce cultural barriers. As reported above, our statistical analysis of the survey results suggested potential generalizability of CDP training emerging from this study, as the greatest (and least) difficulties 
experienced in the shift to virtual services did not differ across any specific demographic characteristics of CDPs. This research offers contextual background and a solid foundation for creating more effective programs and services during the ongoing pandemic and beyond.

Although the focus of this article has been primarily on the challenges experienced in the rapid shift to virtual services, it is also important to highlight the normality and symmetry of the data. This suggests that the career development and employment services sector is not in a dire situation; in fact, the most common response was "No Change" in difficulty across a range of key CDP competencies since moving to virtual services, and about half of responses indicated that those competencies had actually become easier.

However, there are some limitations to the study that must not be ignored. For example, this study focused exclusively on CDPs who deliver services within British Columbia. Therefore, we cannot comment on whether or not the challenges for CDPs that surfaced in our results would be replicated in CDPs from other regions. Many of our participants were also part of ASPECT BC's network; therefore, our sample lacked randomization, which increases the potential of biases within both survey answers and focus group discussions. Finally, due to us still being mid-pandemic at the time of writing this article, there is little complementary research on how the immediate shift to virtual services that accompanied COVID-19 impacted CDPs' ability to effectively use their job-related competencies. As the pandemic continues and we move towards more in-person interactions, further research is needed to explore how work structures may have permanently changed for CDPs; of particular interest may be how hybrid models of work may impact CDP service delivery going forward.

In the short term, this research has already been used to inform work being undertaken by ASPECT BC and its project partner, ETHOS Career Management Group, to create and test educational interventions to address some of the gaps in online competencies that surfaced through this study. Recommendations and interventions will be shared with the original VLC; their consultation will help to ensure that, moving forward, the needs of CDPs are effectively addressed in this community-based project. We hope that this research will serve as a useful jumping off point, illuminating a pathway for educational interventions that will serve to mitigate the challenges that CDPs and their clients face in the virtual world. As it is likely that virtual career development services will continue in some way well after the end of the pandemic, our hope is that this research will continue to inform training and capacity-building for CDPs in BC and beyond, equipping them to continue to meet emerging demands within the workforce development landscape.

\section{References}

Abbott, A. (2021). COVID's mental-health toll: How scientists are tracking a surge in depression. Nature, 590(7845), 194195. https://doi.org/10.1038/ d41586-021-00175-z

Akkermans, J., Richardson, J., \& Kraimer, M. L. (2020). The COVID-190 crisis as a career shock: Implications for careers and vocational behavior. Journal of Vocational Behavior, 119, 103434. https://doi.org/10.1016/j. jvb.2020.103434

Amundson, N. E., Harris-Bowlsbey, J., \& Niles, S. G. (2009). Essential elements of career counseling: Processes and techniques. Saddle River, NJ: Pearson Education.

Banks, S., Cai, T., de Jonge, E., Shears, J., Shum, M., Sobočan, A. M., Strom, K., Truell, R., Úriz, M. J., \& Weinberg, M. (2020). Practising ethically during COVID-19: Social work challenges and responses. International Social Work, 63(5), 569-583. https://doi.org/10.11 77\%2F0020872820949614

Batastini, A. B., Paprzycki, P., Jones, A. C. T., \& MacLean, N. (2021). Are videoconferenced mental and behavioral health services just as good as in-person? A meta-analysis of a fast-growing practice. Clinical Psychology Review, 83, 101944. https://doi.org/10.1016/j. cpr.2020.101944 Canadian Career Development 
Foundation. (2021). Competency_profile_CDP. http:// ccdp-pcdc.ca/en/Competency Profile CDP.pdf

CERIC. (2020). 2019 Survey of Career Service Professionals: Who We Are and Where We're Going. https:// ceric.ca/wp-content/uploads/2020/02/2019-National-Survey-Findings-English. pdf

Chan, C., \& Au-Yueng, H. (2021). When narrative practice suddenly goes online due to COVID-19 .... Qualitative Social Work, 20(1-2), 390-398.h ttps://doi.org/10.1177\%2F147 3325020981086

Como, R., Hambley, L., \& Domen e, J. (2021). An exploration of work-life wellness and remote work during and beyond $\mathrm{COV}$ ID-19. Canadian Journal of C areer Development, 20(1), 4656. https://cjcd-rcdc.ceric.ca/i ndex.php/cjcd/article/view/92

Creswell, J. W., \& Clark, V. L. P. (2017). Designing and condu cting mixed methods research. Sage.

Fortgang, R. G., Wang, S. B., Mill ner, A. J., Reid-Russell, A., B eukenhorst, A. L., Kleiman, E . M., Bentley, K. H., Zuromsk i, K. L., Al-Suwaidi, M., Bird , S. A., Buonopane, R., DeMa rco, D., Haim, A., Joyce, V. W ., Kastman, E. K., Kilbury, E., Lee, H.-I. S., Mair, P., Nash, C . C., ... Nock, M. K. (2021). I ncrease in suicidal thinking du ring COVID-19. Clinical Psyc hological Science, 9(3), 482-4 88. https://doi.org/10.1177\%2 $\underline{\text { F2167702621993857 }}$
Hames, J. L., Bell, D. J., Perez-Li ma, L. M., Holm-Denoma, J. M., Rooney, T., Charles, N. E. , Thompson, S. M., Mehlenbe ck, R. S., Tawfik, S. H., Fond acaro, K. M., Simmons, K. T. , \& Hoersting, R. C. (2020). N avigating uncharted waters: $\mathrm{C}$ onsiderations for training clin ics in the rapid transition to te lepsychology and telesupervi sion during COVID-19. Jour nal of Psychotherapy Integra tion, 30(2), 348-365. https:// psycnet.apa.org/doi/10.1037/ int0000224

Hill, R. M., Rufino, K., Kurian, S. , Saxena, J., Saxena, K., \& Wi lliams, L. (2021). Suicide idea tion and attempts in a pediatri c emergency department befor e and during COVID-19. Pedi atrics, 147(3).https://pubmed. ncbi.nlm.nih.gov/33328339/

Kettunen, J., \& Sampson, J. P. (2 019). Challenges in implem enting ICT in career services : Perspectives from career de velopment experts. Internatio nal Journal for Educational a nd Vocational Guidance, 19 (1), 1-18. https://eric.ed.gov /?id=EJ1211572

King-Nyberg, B., Thomson, E., F. , Morris-Reade, J., Taylor, C. , \& Borgen, R., A. (2021). A b ot toolbox: An accidental cas e study on how to eliminate bo ts from your online survey. $\mathrm{M}$ anuscript submitted for publi cation.

Luken, T. (2019, October 23). Bi $\mathrm{g}$ problem in career counselli ng- and a possible way out. $\mathrm{C}$ areerWise/CERIC. https://careerwise.ceric.ca/2019/10/23/ big-problems-in-career-counselling-and-a-possible-wayout/\#.YemffnrMJhE

Organisation for Economic Co-op eration and Development. (20 20, April 28). Public employm ent services in the frontline fo r jobseekers, workers and em ployers. OECD. https://www. oecd.org/coronavirus/policy-responses/public-employment-services-in-the-frontline-for-employees-jobseekers-and-employers-c986ff92/

Panchal, N., Kamal, R., Cox, C., G arfield, R. (2021, February 10 ). The implications of COVID -19 for mental health and sub stance use. KFF. https://www. kff.org/coronavirus-covid-19/ issue-brief/the-implicationsof-covid-19-for-mentalhealth-and-substance-use/ Redekopp, D. E., \& Huston, M. (2 019). The broader aims of car eer development: Mental healt $\mathrm{h}$, wellbeing and work. British Journal of Guidance \& Couns elling, 47(2), 246-257. https:// doi.org/10.1080/03069885.20 $\underline{18.1513451}$

Sampson, J., Kettunen, J., \& Vu orinen, R. (2020). The role o f practitioners in helping per sons make effective use of in formation and communicatio $\mathrm{n}$ technology in career interv entions. International Journa I for Educational and Vocati onal Guidance, 20, 191-208. https://www.researchgate.net/ publication/334096445 The role of practitioners in helping persons make effective use of information and communication technol- 
ogy_in_career_interventions

Schlossberg, N. K., Lunch, A. Q., \& Chickering, A. W. (1989). I mproving higher education e nvironments for adults. Josse $\mathrm{y}$-Bass.

Neault, R. A. (2002). Thriving in $t$ he new millennium: Career $\mathrm{m}$ anagement in the changing wo rld of work. Canadian Journa l of Career Development, 1(1) , 11-21.

Neault, R. A., \& Pickerell, D. A. ( 2013). Skill requirements for BCs' career practitioners: A $n$ exploratory study. BC Cen tre for Employment Excellen ce. https://www.cfeebc.org/ skill-requirements-for-bcs-career-development-practitioners/

Walters, B., Shepard, B., \& Mani , P. (2014). Developing effecti ve client relationships. In B. S hepard \& P. Mani (Eds.), Care er development practice in $\mathrm{Ca}$ nada: Perspectives, principle $s$, and professionalism (pp. 15 3-167). CERIC.

World Economic Forum. (2020). The future of jobs report (pp. 9-35). https://www.weforum. org/reports/the-future-of-jobsreport-2020

Yukl, G. (2006). Leadership in org anizations (6th ed.). Pearson $\mathrm{P}$ rentice. 